Mathematical Research Letters 9, 73-93 (2002)

\title{
ANALYTIC REGULARITY OF CR-MAPPINGS
}

\author{
Francine Meylan, Nordine Mir and Dmitri Zaitsev
}

\section{Introduction}

In this paper we give general conditions that guarantee the analyticity of $\mathcal{C}^{\infty}$-smooth CR-mappings between real-analytic CR-submanifolds $M \subset \mathbb{C}^{N}$ and $M^{\prime} \subset \mathbb{C}^{N^{\prime}}$ (see Theorem 1.1). The proof of the analyticity is based on results on holomorphic and meromorphic extension of functions on wedge-like domains to boundary points (see Theorems 2.4 and 2.6) that may be of independent interest.

The analyticity problem for CR-mappings $f: M \rightarrow M^{\prime}$ between real-analytic CR-submanifolds goes back to the work of LEWY [Le56, Le77] and PINCHUK [Pi75], where the analyticity of a CR-diffeomorphism $f$ was shown for $M, M^{\prime} \subset$ $\mathbb{C}^{N}$ Levi-nondegenerate hypersurfaces. The condition of Levi-nondegeneracy of $M$ (resp. of $M^{\prime}$ ) can be seen as a nonvanishing condition involving first and second order partial derivatives of a local defining function $\rho(z, \bar{z})$ of $M$ (resp. $\rho^{\prime}\left(z^{\prime}, \overline{z^{\prime}}\right)$ of $\left.M^{\prime}\right)$. Motivated by this and other conditions due to the work of other authors (see below), one may pose the analyticity problem in the following more precise form:

(*) Find open conditions, each involving finitely many derivatives of $f$ and of $\rho$ and $\rho^{\prime}$ at the reference points which imply, in the above setting, that $f$ is real-analytic.

Here, by an open condition, we mean a condition on finitely many derivatives that holds on an open subset in the space of derivatives. For instance, the condition of minimality (which is equivalent to that of finite type in the sense of KoHN and BLOOM-GRAHAM for a real-analytic CR-manifold $M$ ), as well as that of essential finiteness of $M$ (see e.g. [BER99]), can be expressed as open conditions on the derivatives of $\rho$. The reader is referred to the papers [W78, DW80, W82, Ha83, CKS84, BJT85, BBR88, BR88, BP88, DF88, Fo89, BR90b, Fa90, Su92, DFY94, Hu94, DP95, Me95, Hu96, BHR95, HP96, Ha97, CPS99, BER00, La01] containing as main results analyticity statements for CRmappings under open conditions in the above sense. In a different direction, the analytic regularity of CR-mappings has been studied by many authors under other types of conditions, for instance assuming a priori holomorphic extension of $f$ to a wedge. Other results assume weak pseudoconvexity that is, in contrast

Received June 19, 2001.

2000 Mathematics Subject Classification. 32C16, 32H02, 32H40, 32V20, 32V25.

The first author was partially supported by Swiss NSF Grant 2100-063464.00/1 . 
to strong pseudoconvexity, not an open condition in the above sense (see also [E00] for another kind of such non-open condition). These results are beyond the scope of the present work.

In this paper we prove a new analyticity result for CR-mappings between realanalytic CR-submanifolds, of any CR dimension, in complex spaces of arbitrary dimension. The analyticity is proved for a source manifold $M$ minimal and under a condition that holds, to the authors' knowledge, in all previous works in the context of $(*)$.

To formulate our result, consider the family of Segre varieties associated to $M$ and $M^{\prime}$ given by

$$
Q_{w}:=\{z: \rho(z, \bar{w})=0\}, \quad Q_{w^{\prime}}^{\prime}:=\left\{z^{\prime}: \rho^{\prime}\left(z^{\prime}, \overline{w^{\prime}}\right)=0\right\},
$$

for $w$ and $w^{\prime}$ near the reference points $p \in M$ and $p^{\prime} \in M^{\prime}$ respectively, where $\rho(z, \bar{z})$ and $\rho^{\prime}\left(z^{\prime}, \overline{z^{\prime}}\right)$ are any real-analytic (vector-valued) defining functions of $M$ and $M^{\prime}$ near $p$ and $p^{\prime}$ respectively. Given a $\mathcal{C}^{\infty}$-smooth CR-mapping $f: M \rightarrow M^{\prime}$ with $f(p)=p^{\prime}$, it is known (see e.g. [BER99, Proposition 1.7.14]) that the Taylor series of $f$ at $p$ extends to a formal (holomorphic) power series mapping $F_{p}:\left(\mathbb{C}^{N}, p\right) \rightarrow\left(\mathbb{C}^{N^{\prime}}, p^{\prime}\right)$ sending (formally) $Q_{p}$ into $Q_{p^{\prime}}^{\prime}$. Define a germ of a complex-analytic subset of $\mathbb{C}^{N^{\prime}}$ through $p^{\prime}$ by

$$
r_{p}:=\left\{w^{\prime}: Q_{w^{\prime}}^{\prime} \supset F_{p}\left(Q_{p}\right)\right\},
$$

where the inclusion is understood in the sense of formal power series (see $\S 3$ for more details). Subsets similar to $r_{p}$ have been considered by many authors (see e.g. [DW80, BJT85, BR88, DF88, Fo89, DP95, Hu96, CPS99, Z99, Mi00]).

The main result of this paper is the following.

Theorem 1.1. Let $M \subset \mathbb{C}^{N}, M^{\prime} \subset \mathbb{C}^{N^{\prime}}$ be real-analytic CR-submanifolds and $f: M \rightarrow M^{\prime}$ a $\mathcal{C}^{\infty}$-smooth CR-mapping. Assume that $M$ is minimal at a point $p \in M$ and that $p^{\prime}=f(p)$ is isolated in $r_{p}$. Then $f$ extends holomorphically to a neighborhood of $p$ in $\mathbb{C}^{N}$.

The equivalence of the analyticity of $f$ and its holomorphic extension is a consequence of a theorem of Tomassini [To66]. In the case when $M \subset \mathbb{C}^{N}$ is a real hypersurface, Theorem 1.1 is due to Coupet-Pinchuk-Sukhov [CPS99]. One additional difficulty for a source manifold $M$ of higher codimension is that a one-sided extension for CR-functions on $M$ has to be replaced by a wedge extension, where the additional directions of the wedges may change as $M$ is shrinked (see [BGN98] and Example 4.1 below). As a consequence, we need to establish Theorem 2.4 and 2.6 below whose proof requires the full machinery of Tumanov's CR-extension theory.

The condition in Theorem 1.1 plays an important role also in different questions. For instance, under the same assumptions on $M$ and $M^{\prime}$, the second author showed that a formal mapping is always convergent [Mi00, Theorem 9.1].

Remark 1.2. The statement of Theorem 1.1 holds (with the same proof) when the target $M^{\prime}$ is any real-analytic subset of $\mathbb{C}^{N^{\prime}}$. 
Observe that the condition that $p^{\prime}$ is isolated in $r_{p}$ in Theorem 1.1 involves all the data $M, M^{\prime}$ and $f$. It may be of interest to give independent conditions on $M, M^{\prime}$ and $f$. We conclude by giving a corollary of Theorem 1.1 assuming separate conditions on $M, M^{\prime}$ and $f$. For this, recall that $M^{\prime}$ is called essentially finite (see [DW80, BJT85]) at a point $p^{\prime} \in M^{\prime}$ if $p^{\prime}$ is isolated in the set $\left\{w^{\prime}\right.$ : $\left.Q_{w^{\prime}}^{\prime} \supset Q_{p^{\prime}}^{\prime}\right\}$. Furthermore, we say that $f$ is not totally degenerate at $p$ if the (generic) rank of the formal map between $Q_{p}$ and $Q_{p^{\prime}}^{\prime}$ induced by the above map $F_{p}$ equals the dimension of $Q_{p^{\prime}}^{\prime}$ (see $\S 3$ for more details).

Corollary 1.3. In the setting of Theorem 1.1, suppose that $M$ is minimal at a point $p \in M, M^{\prime}$ is essentially finite at $p^{\prime}=f(p)$ and $f$ is not totally degenerate at $p$. Then $f$ extends holomorphically to a neighborhood of $p$ in $\mathbb{C}^{N}$.

In the case $N=N^{\prime}, \operatorname{dim} M=\operatorname{dim} M^{\prime}$ and $\operatorname{dim} Q_{p}=\operatorname{dim} Q_{p^{\prime}}^{\prime}$, Corollary 1.3 was obtained by BAOUEndi-RothschiLd [BR90b] when $M$ and $M^{\prime}$ are hypersurfaces and by the first author [Me95] in higher codimension. We would like to point out that, even in the case when $M, M^{\prime} \subset \mathbb{C}^{N}$ are real hypersurfaces of the same dimension, the situation considered in Theorem 1.1 is more general than that in Corollary 1.3 as will be illustrated by Example 6.5.

The paper is organized as follows. In $\S 2$ we present the statements of the main tools needed for the proof of Theorem 1.1. In $\S 3$ we introduce and explain the necessary notation and terminology needed throughout the paper. In $\S 4$ we study holomorphic hulls of wedges, introduce the notion of generalized wedge and prove Theorem 2.4. $\S 5$ is devoted to the proof of Theorem 2.6 obtained by combining Theorem 2.4, a separate meromorphicity result due to SHIFFMAN and a result due to IVASHKOVICH concerning envelopes of holomorphy and meromorphy. Finally in $\S 6$ we prove Theorem 1.1 and show how Corollary 1.3 can be derived from it.

\section{Main tools}

We begin with the case of real hypersurfaces in $\mathbb{C}^{n}$ which is essentially simpler than that of higher codimension. An important phenomenon in several complex variables is the "forced" extension of holomorphic functions from one side of a real hypersurface $M \subset \mathbb{C}^{N}$ to the other side. For strongly pseudoconcave sides, such an extension is a consequence of the classical Hartogs theorem. More generally, by a theorem of TréPreAu [Tr86] (see also BAOUEnd-Treves [BT84] for $M$ real-analytic), such an extension holds for at least one of the two sides if and only if $M$ does not contain complex hypersurfaces. As a corollary, one has holomorphic extension of CR-functions on $M$ to the same side.

A similar phenomenon for generic submanifolds $M \subset \mathbb{C}^{N}$ of higher codimension is much less understood and is one of the main subjects of the present paper. Here the natural substitutes for one-sided neighborhoods are wedges with edge $M$ and the necessary and sufficient condition for holomorphic extension of CRfunctions on $M$ to such a wedge is the minimality (see $\S 3$ ) due to the theorems of Tumanov [Tu88] and of Baouendi-Rothschild [BR90a]. 
A significant difficulty when working with wedge extensions instead of onesided extensions is due to the fact that the normal directions of the wedges, where the extension takes place, depend not only on the local geometry of $M$ but also on the size of $M$ near a given point as e.g. an example of BoGGESS-GLENNNagel [BGN98] (see Example 4.1) shows. This difficulty becomes visible when studying extension properties of CR-mappings between real-analytic generic submanifolds. Here the reflection principle yields natural reflections associated to real-analytic submanifolds going back to the Schwarz reflection in one complex variable. More generally, we define:

Definition 2.1. Let $M \subset \mathbb{R}^{n}$ be a real submanifold of class $\mathcal{C}^{k}, k=1, \ldots, \infty$. A reflection with respect to $M$ is a diffeomorphism $\nu: \omega \rightarrow \omega$ of class $\mathcal{C}^{k}$, where $\omega$ is a neighborhood of $M$ in $\mathbb{R}^{n}$, such that $\left.\nu\right|_{M}=\mathrm{id}$ and the induced self-map of the normal space $T_{q} \mathbb{R}^{n} / T_{q} M$ equals -id for each $q \in M$.

If $M$ and $\omega$ are as above, any involution $\nu: \omega \rightarrow \omega$ for which the fixed point set is $M$ is a reflection as in Definition 2.1. Furthermore, for a real hypersurface $M$, any reflection locally exchanges the sides of $M$. Motivated by this case, we are led to study the following question:

If $M$ is minimal at a point $p \in M$ and $\nu: \omega \rightarrow \omega$ is a reflection with respect to $M$, is there a wedge $\mathcal{W} \subset \omega$ with edge $M$ such that

(i) all CR-functions on $M$ extend holomorphically to $\mathcal{W}$;

(ii) all holomorphic functions on $\nu(\mathcal{W})$ extend holomorphically to a fixed neighborhood of $p$ ?

It turns out that, for wedges in the classical sense obtained by adding transversal cones to neighborhoods of $p$ in $M$, the answer to the above question is negative. In fact we show that Example 4.1 provides a counterexample. However, the answer becomes positive if one allows $\mathcal{W}$ to have variable normal directions. Such $\mathcal{W}$ will be called a generalized wedge and is defined as follows:

Definition 2.2. Let $M \subset \mathbb{R}^{n}$ be a real submanifold through a point $p \in M$ and $\Pi \subset \mathbb{R}^{n}$ a linear subspace with $T_{p} M \oplus \Pi=\mathbb{R}^{n}$. A generalized wedge with edge $M$ at $p$ (relative to $\Pi$ ) is a connected open subset $\mathcal{W} \subset \mathbb{R}^{n}$ of the form

$$
\mathcal{W}=\left\{x+y: x \in U, y \in \Gamma_{x} \cap \Omega\right\},
$$

where $U \subset M$ is an open neighborhood of $p, \Gamma_{x} \subset \Pi$ is an open convex cone with vertex the origin for each $x \in U$ and $\Omega \subset \Pi$ is an open neighborhood of 0 . We call $U$ the base of $\mathcal{W}$. In the classical case when $\Gamma_{x}=\Gamma \subset \Pi$ is independent of $x \in U$, we call $\mathcal{W}$ a standard wedge (in the direction $\Gamma$ ) and write $\mathcal{W}=W(U, \Omega, \Gamma)$.

Remark 2.3. Note that if $\mathcal{W}$ is a generalized wedge as in Definition 2.2, then there exists $\epsilon>0$ and a $\mathcal{C}^{\infty}$-smooth mapping $U \ni x \mapsto v_{x} \in \Gamma_{x} \subset \Pi$ such that for any point $x \in U$, there exists a (standard) wedge $W_{x} \subset \mathcal{W}$ with edge $M$ at $x$ with $x+t v_{x} \in W_{x}$ for $t \in \mathbb{R},|t|<\epsilon$. 
We have the following result answering positively the above question for generalized wedges:

Theorem 2.4. Let $M \subset \mathbb{C}^{N} \cong \mathbb{R}^{2 N}$ be a generic submanifold of class $\mathcal{C}^{2, \alpha}$ which is minimal at a point $p \in M$. Fix $\Pi \subset \mathbb{R}^{2 N}$ as in Definition 2.2. Then for any sufficiently small open neighborhood $U$ of $p$ in $M$, there exists a generalized wedge $\mathcal{W}$ with edge $M$ at $p$ with base $U$ satisfying the following properties:

(i) all continuous $C R$-functions on $M$ extend holomorphically to $\mathcal{W}$;

(ii) for any reflection $\nu: \omega \rightarrow \omega$ with respect to $M$, all holomorphic functions on $\nu(\mathcal{W} \cap \omega)$ extend holomorphically to a fixed neighborhood of $p$ in $\mathbb{C}^{N}$.

As will be explained in $\S 4$, the statement of Theorem 2.4 does not hold for standard wedges instead of generalized wedges. Theorem 2.4 will be one of the main steps in proving the analyticity result for CR-mappings provided by Theorem 1.1. The authors know of no other examples of an analyticity result of this kind whose proof requires the use of generalized wedges instead of standard ones.

Property (ii) in Theorem 2.4 expresses the fact that the envelope of holomorphy of the open subset $\nu(\mathcal{W} \cap \omega)$ contains a neighborhood of $p$ in $\mathbb{C}^{N}$. Together with a theorem of Ivashkovich [I92], we obtain:

Corollary 2.5. In the setting and with the notation of Theorem 2.4, all meromorphic functions on $\nu(\mathcal{W} \cap \omega)$ extend meromorphically to a fixed neighborhood of $p$ in $\mathbb{C}^{N}$.

As an application of Corollary 2.5, we shall prove in $\S 5$ the following result on meromorphic extension:

Theorem 2.6. Let $U \subset \mathbb{C}^{N}, V \subset \mathbb{C}^{m}$ be open subsets, $M \subset U$ a connected generic real-analytic submanifold, $g: M \rightarrow V$ a continuous $C R$-function and $u, v: U \times V^{*} \rightarrow \mathbb{C}$ holomorphic functions, where $V^{*}:=\{\bar{z}: z \in V\}$. Assume that $M$ is minimal at every point and that there exists a nonempty open subset of $M$, where $v(z, \overline{g(z)})$ does not vanish and where the quotient

$$
\frac{u(z, \overline{g(z)})}{v(z, \overline{g(z)})}
$$

is $C R$. Then $v(z, \overline{g(z)})$ does not vanish on a dense open subset $M_{0} \subset M$ and the quotient (2.1) extends from $M_{0}$ meromorphically to a neighborhood of $M$ in $\mathbb{C}^{N}$.

In the case when $M$ is a real hypersurface, analogous results were obtained by BAOUENDI-HuAng-Rothschild [BHR96] in an algebraic context and later by Coupet-Pinchuk-Sukhov [CPS99] for $M$ real-analytic (see also [Pu90a, Pu90b, CPS00]). In each of these papers the corresponding meromorphic extension result was an important step in proving analyticity of CR-mappings. Theorem 2.6 in the hypersurface case is stronger than the corresponding results in the two papers mentioned above. Indeed, the quotient (2.1) is required here to be CR only on an open subset instead of an open dense subset and $g$ is only 
required to be continuous. Another application of Theorem 2.6 to the analyticity of CR-mappings will be given in the forthcoming paper of the authors [MMZ01].

\section{Preliminaries}

We say that a submanifold $M \subset \mathbb{R}^{n}$ is of class $\mathcal{C}^{k, \alpha}, k \geq 1,0<\alpha<1$, if, near each of its points, $M$ has a set of defining functions of class $\mathcal{C}^{k}$ with $k$-th order partial derivatives satisfying a Hölder condition with exponent $\alpha$. Recall that $M$ is called a $C R$-submanifold if the dimension of the complex tangent space $T_{q}^{c} M:=T_{q} M \cap i T_{q} M$ is independent of $q \in M ; M$ is called generic if $\operatorname{dim}_{\mathbb{C}} T_{q}^{c} M=\operatorname{dim}_{\mathbb{R}} M-N$ for all $q \in M$. Recall also that a CR-submanifold $M$ is called minimal (see [Tu88]) at a point $p \in M$ if there is no real submanifold $S \subset M$ through $p$ with $\operatorname{dim} S<\operatorname{dim} M$ such that $T_{q}^{c} M \subset T_{q} S$ for all $q \in S$.

A continuous function on $M$ is called a $C R$-function if it is annihilated, in the sense of distributions, by the $\mathrm{CR}$ vector fields of $M$ (i.e. $(0,1)$ vector fields tangent to $M)$. A continuous mapping between two CR-submanifolds $M \subset \mathbb{C}^{N}$ and $M^{\prime} \subset \mathbb{C}^{N^{\prime}}$ is called a $C R$-mapping if all its components are CR-functions.

Let $X \subset \mathbb{C}^{N}$ and $X^{\prime} \subset \mathbb{C}^{N^{\prime}}$ be complex-analytic submanifolds through $p \in$ $\mathbb{C}^{N}$ and $p^{\prime} \in \mathbb{C}^{N^{\prime}}$ respectively. We say that a formal (holomorphic) power series $h \in \mathbb{C}[[z-p]], z=\left(z_{1}, \ldots, z_{N}\right)$, vanishes on $X$ if $h \circ v=0$ for one (and hence for any) complex-analytic parametrization $v:\left(\mathbb{C}^{\operatorname{dim} X}, 0\right) \rightarrow(X, p)$ of $X$ near $p$. We further say that a formal power series mapping $F:\left(\mathbb{C}^{N}, p\right) \rightarrow\left(\mathbb{C}^{N^{\prime}}, p^{\prime}\right)$ sends $X$ into $X^{\prime}$ and write $F(X) \subset X^{\prime}$ if, for any formal power series $h^{\prime} \in \mathbb{C}\left[\left[z^{\prime}-p^{\prime}\right]\right]$ vanishing on $X^{\prime}, z^{\prime}=\left(z_{1}^{\prime}, \ldots, z_{N^{\prime}}^{\prime}\right)$, the composition $h^{\prime} \circ F$ vanishes on $X$. In this case the map $F$ induces a formal power series mapping $\left.F\right|_{X}$ between $X$ and $X^{\prime}$. Then, by the (generic) rank of $\left.F\right|_{X}$ we mean the largest integer $r$ such that some $r \times r$ minor of the Jacobian matrix of $F \circ v$, where $v$ is as above, does not vanish identically.

We give here more precise details on the complex-analytic set $r_{p}$ defined in (1.2). Let $M \subset \mathbb{C}^{N}$ and $M^{\prime} \subset \mathbb{C}^{N^{\prime}}$ be real-analytic CR-submanifolds through points $p$ and $p^{\prime}$ respectively. Let $\rho(z, \bar{z}), \rho^{\prime}\left(z^{\prime}, \overline{z^{\prime}}\right)$ be vector-valued local realanalytic defining functions for $M$ near $p$ and $M^{\prime}$ near $p^{\prime}$ respectively, with complex differentials of constant rank. For sufficiently small neighborhoods $U \subset \mathbb{C}^{N}, U^{\prime} \subset \mathbb{C}^{N^{\prime}}$ of $p$ and $p^{\prime}$, the family of Segre varieties of $M$ and $M^{\prime}$ is the family of complex submanifolds given by $Q_{w}=\{z \in U: \rho(z, \bar{w})=0\}$ and $Q_{w^{\prime}}^{\prime}=\left\{z^{\prime} \in U^{\prime}: \rho^{\prime}\left(z^{\prime}, \overline{w^{\prime}}\right)=0\right\}$ for $w$ and $w^{\prime}$ sufficiently close to $p$ and $p^{\prime}$ respectively. Given a $\mathcal{C}^{\infty}$-smooth CR-map $f: M \rightarrow M^{\prime}$ with $f(p)=p^{\prime}$, we denote, as in $\S 1$, by $F_{p}$ a $\mathbb{C}^{N^{\prime}}$-valued formal (holomorphic) power series centered at $p$ which extends the Taylor series of $f$ at $p$. (Note that if $M$ is generic, such a formal power series mapping is unique.) Choose a holomorphic parametrization $\gamma=\gamma(t)$ of the Segre variety $Q_{p}, t=\left(t_{1}, \ldots, t_{n}\right)$, with $n=\operatorname{dim}_{\mathbb{R}} T_{p}^{c} M$ and $\gamma(0)=p$. Then, for a point $w^{\prime}$ close to $p^{\prime}$, the formal inclusion $Q_{w^{\prime}}^{\prime} \supset F_{p}\left(Q_{p}\right)$ is equivalent to the identity $\rho^{\prime}\left(F_{p}(\gamma(t)), \overline{w^{\prime}}\right) \equiv 0$ in the ring $\mathbb{C}[[t]]$. From this, we see that (1.2) defines a germ of a complex-analytic set through the point $p^{\prime}$. It 
is also easy to see that the set $r_{p}$ is independent of the choice of $F_{p}, \gamma$ and local vector-valued defining functions for $M$ and $M^{\prime}$.

\section{Holomorphic extension of functions on wedges; proof of Theorem 2.4}

In this section we shall prove Theorem 2.4. Given a generic submanifold $M \subset \mathbb{C}^{N}$ as in Theorem 2.4 which is minimal at a point $p$, a natural attempt would be to apply TumANOV's theorem [Tu88] to get a standard wedge $W$ with edge $M$ at $p$ satisfying property (i) of that theorem. Then, given any reflection $\nu$ with respect to $M$ (as in Definition 2.1), in order to show (ii), one can try to "push" the manifold $M$ a little to get a submanifold $\widetilde{M}$ inside $\nu(W)$. Then any holomorphic function on $\nu(W)$ would restrict to a CR-function on $\widetilde{M}$ which, in turn, would extend to the "pushed wedge". This last wedge would contain a neighborhood of $p$ because it is "pushed" in the opposite direction with respect to that of $W$.

However, this simple attempt leads to the following problem: in general, the base $U \subset M$ of the wedge $W$, as in Definition 2.2, is strictly smaller than $M$. Hence the reflected wedge $\nu(W)$ will not contain the "pushed" manifold $\widetilde{M}$ but only the "pushed" base $\widetilde{U}$ or even a smaller domain. Of course, one may use TUMANOV's theorem again for the submanifold $\widetilde{U}$ to get a new standard wedge $W_{1}$. However, the wedge $W_{1}$ may not have any direction that is opposite to any direction of $\nu(W)$ and thus $W_{1}$, when "pushed" into $\nu(W)$, may not cover any neighborhood of $p$.

This problem does not appear if $M \subset \mathbb{C}^{N}$ is a real hypersurface. In that case, by applying TRÉPREAU's theorem [Tr86], one finds a base of neighborhoods of $p$ in $M$ such that the holomorphic extension takes place to the same side of $M$ for all neighborhoods. Therefore any smaller one-sided neighborhood with holomorphic extension can be chosen to be opposite to that of $\nu(W)$ and, hence, the above argument applies.

In contrast to the hypersurface case, if $M \subset \mathbb{C}^{N}$ is a generic submanifold of higher codimension which is minimal at $p$, the direction of a wedge of holomorphic extension may change when shrinking the base $U$. This phenomenon is illustrated on the following example.

Example 4.1. Consider the generic submanifold $M \subset \mathbb{C}^{3}$ of codimension 2 given by

$$
M:=\left\{\left(z, w_{1}, w_{2}\right) \in \mathbb{C}^{3}: \operatorname{Im} w_{1}=|z|^{2}, \operatorname{Im} w_{2}=|z|^{4}\right\} .
$$

The neighborhood

$$
U_{\varepsilon}:=\left\{\left(z, w_{1}, w_{2}\right) \in M:|z|<\varepsilon\right\}
$$

of 0 in $M$ is contained in the closure of the convex tube domain $V_{\varepsilon}$ given by

$$
V_{\varepsilon}:=\left\{\left(z, w_{1}, w_{2}\right) \in \mathbb{C}^{3}:\left(\operatorname{Im} w_{1}\right)^{2}<\operatorname{Im} w_{2}<\varepsilon^{2} \operatorname{Im} w_{1}\right\} .
$$


Hence any wedge $W_{\varepsilon}$ with edge $U_{\varepsilon}$ at 0 , where all CR-functions on $U_{\varepsilon}$ holomorphically extend, must be contained in $V_{\varepsilon}$. Indeed, otherwise one has a CRfunction on $U_{\varepsilon}$ of the form $f(z)=1 /(\lambda(z)-c)$, where $\lambda$ is a complex-linear form and $c$ is a constant, that does not extend to the whole $W_{\varepsilon}$. Then, given any such $W_{\varepsilon_{0}}$ for $U_{\varepsilon_{0}}$, if $\varepsilon_{1}>0$ is sufficiently small, any wedge $W_{\varepsilon_{1}} \subset V_{\varepsilon_{1}}$ will not share any direction with $W_{\varepsilon_{0}}$.

Furthermore, we claim that any neighborhood $M_{0}$ of 0 in $M$ yields a counterexample to the statement of Theorem 2.4 with "generalized wedge" replaced by "standard wedge". Indeed, set

$$
\varepsilon:=\sup \left\{|z|:\left(z, w_{1}, w_{2}\right) \in M_{0} \text { for some } w_{1}, w_{2}\right\} \leq \infty
$$

and suppose that a standard wedge $\mathcal{W}=W(U, \Omega, \Gamma)$ satisfies the conclusion of Theorem 2.4. Then $U$ is contained in $U_{\varepsilon}$ and property (i) in Theorem 2.4 together with the above remark implies that $\mathcal{W}$ is contained in $V_{\varepsilon}$. Hence the convex cone $\Gamma$ is of the form $\left\{\left(v_{1}, v_{2}\right) \in \mathbb{R}^{2}: \alpha^{2} v_{1}<v_{2}<\beta^{2} v_{1}\right\}$ for some $0<\alpha<\beta \leq \varepsilon$. Since $x+(\Gamma \cap \Omega) \subset \mathcal{W} \subset V_{\varepsilon}$ holds for any $x \in U \subset M$, it follows that $U \subset U_{\alpha}$ (as in (4.1)) and therefore $U$ is contained in the closure of the convex domain

$$
D:=\left\{\left(z, w_{1}, w_{2}\right) \in \mathbb{C}^{3}: \operatorname{Im} w_{2}<\alpha^{2} \operatorname{Im} w_{1}\right\} .
$$

Finally we choose the reflection $\nu$ to be

$$
\nu:\left(z, w_{1}, w_{2}\right) \mapsto\left(z, \bar{w}_{1}+2 i|z|^{2}, \bar{w}_{2}+2 i|z|^{4}\right) .
$$

Then it is easy to see that $\nu(\mathcal{W})$ is also contained in the domain of holomorphy $D$ and hence property (ii) in Theorem 2.4 cannot hold.

The above mentioned problem is exactly the reason for us to state Theorem 2.4 for a generalized wedge instead of a standard one. We apply TumANOV's theorem twice: first to the generic submanifold $M$ to get a wedge $W_{1}$ with a base $U \subset M$ and then to a smaller neighborhood $U^{\prime} \subset \subset U$ to get another wedge $W_{2}$. We then use a theorem of AJRAPETIAN-HENkin [AH81] to "connect" $W_{1}$ and $W_{2}$ through a third wedge $W_{3}$. The generalized wedge $\mathcal{W}$ is then chosen to be contained in the union of $W_{1}, W_{2}$ and $W_{3}$. Following the above strategy, given any reflection $\nu$ with respect to $M$, we "push" $U^{\prime}$ inside $\nu(\mathcal{W})$. However, in order to get the opposite direction near $p$, we have to push $U^{\prime}$ along a nonconstant transversal vector field. The fact that $U^{\prime}$ is "deformed" along a nonconstant vector field makes it impossible to use the statement of TuMANOV's theorem [Tu88, Tu90] directly, since the statement does not contain any information about the deformation of the wedge $W_{2}$ when the edge is deformed. In order to get control of the size and of the direction of the "deformed wedge" $W_{2}$, we have to use Tumanov's explicit construction of $W_{2}$ based on the method of analytic discs and solving Bishop's equation.

In the explicit construction of Tumanov's wedge, we follow his approach in [Tu96]. The construction consists of two steps. The first one is to construct $d$ analytic discs attached to $M$ through $p$ whose directions spans the normal space 
to $M$ at $p$, where $d$ is the codimension of $M$. Recall that an analytic disc in $\mathbb{C}^{N}$ attached to $M$ is a continuous map $A: \bar{\Delta} \rightarrow \mathbb{C}^{N}$, where $\Delta \subset \mathbb{C}$ is the unit disc, such that $A$ is holomorphic in $\Delta$ and $A(\partial \Delta) \subset M$. Each disc $A_{j}, j=1, \ldots, d$, is constructed such that $A_{j}(1)=p$ and $\partial_{\nu} A_{j}(1) \notin T_{p} M$, where $\partial_{\nu}$ is the radial derivative. Then, each $A_{j}$ can be deformed with $A_{j}(1)$ moving in a neighborhood of $p$ in $M$ to fill a submanifold $M_{j}^{\prime}$ with boundary $M$. In fact, the submanifold $M_{j}^{\prime}$ is filled by pieces of radii of analytic discs of the form $A((1-\varepsilon, 1]), \varepsilon>0$. The second step is an application of a theorem of AJRAPETIAN-HENKIN. It implies that any CR-function on the union of the $M_{j}^{\prime}$ 's extends holomorphically to a wedge $W$. Again, we have to use the explicit construction in order to get the control of $W$ under deformations of the $M_{j}$ 's. The construction of $W$ is also given in [Tu96], where $W$ is obtained as a union of centers of analytic discs attached to $\cup_{j=1}^{d} M_{j}^{\prime}$. The control of the analytic discs entails the control of the wedge $W$. Such a control is based on the solution of Bishop's equation and its regularity with respect to parameters is obtained by TuMANOV in [Tu93].

We now state deformation versions of the CR-extension results from [Tu96] that we shall need for the proof of Theorem 2.4. Recall that a map $\varphi: S \rightarrow \mathbb{R}^{m}$, where $S \subset \mathbb{R}^{n}$, is in the Hölder space $\mathcal{C}^{\alpha}(0<\alpha<1)$ if there exists a constant $C>0$ such that for all $x, y \in S,|\varphi(x)-\varphi(y)| \leq C|x-y|^{\alpha}$. The map $\varphi$ is of class $\mathcal{C}^{k, \alpha}(k \geq 1)$ if it extends to be $\mathcal{C}^{k}$ to a neighborhood of $S$ in $\mathbb{R}^{n}$ such that each partial derivative of $\varphi$ up to order $k$ is of class $\mathcal{C}^{\alpha}$. When considering analytic discs $A: \bar{\Delta} \rightarrow \mathbb{C}^{N}$, it is convenient to assume that they belong to the Hölder spaces $\mathcal{C}^{k, \alpha}$, since such discs are preserved by the Hilbert transform (see e.g. [BER99]). In what follows, given an analytic disc $A: \bar{\Delta} \rightarrow \mathbb{C}^{N}$ of class $\mathcal{C}^{k, \alpha}$, by a small analytic disc we mean an analytic disc with small $\mathcal{C}^{k, \alpha}$ norm.

We say that a family $\left(M_{t}\right)_{t \in T}$, where $T \subset \mathbb{R}$ is an interval, of real submanifolds in $\mathbb{C}^{N}$ is of class $\mathcal{C}^{k, \alpha}$ if near each point there exists a joint local parametrization $x \mapsto \varphi_{t}(x) \in M_{t}$ which is $\mathcal{C}^{k, \alpha}$ with respect to $(t, x)$. In particular, for each $t \in T$, the submanifold $M_{t}$ is of class $\mathcal{C}^{k, \alpha}$. In a similar fashion, one may define a family of submanifolds with boundaries of class $\mathcal{C}^{k, \alpha}$. Note that, if a submanifold $M \subset \mathbb{C}^{N}$ is generic, any submanifold with boundary $M$ is automatically generic. Similarly to wedges we say that $M^{\prime}$ is a submanifold with boundary $M$ at $p \in M$ if the boundary of $M^{\prime}$ is an open neighborhood of $p$ in $M$. The following fact is a deformation version of Lemma 6.4 in [Tu96].

Proposition 4.2. Given a generic submanifold $M \subset \mathbb{C}^{N}$ of class $\mathcal{C}^{k, \alpha}(k \geq$ $1,0<\alpha<1)$ through 0 and a sufficiently small analytic disc $A_{0}: \bar{\Delta} \rightarrow \mathbb{C}^{N}$ of class $\mathcal{C}^{k, \alpha}$ with $A_{0}(1)=0$ and $\xi:=\partial_{\nu} A_{0}(1) \notin T_{0} M$, the following hold:

(i) For any $0<\beta<\alpha$, there exists a (generic) submanifold $M^{\prime}$ of class $\mathcal{C}^{k, \beta}$ with boundary $M$ at 0 such that $T_{0} M^{\prime}=\mathbb{R} \xi \oplus T_{0} M$ and all continuous $C R$-functions on $M$ extend to be $C R$ on $M^{\prime}$.

(ii) Moreover, the submanifold $M^{\prime}$ given in (i) can be chosen with the following property. Given a $\mathcal{C}^{k, \alpha}$-family $\left(M_{t}\right)_{0 \leq t \leq \varepsilon}$ of generic submanifolds with $M_{0}=M$ and given any $0<\beta<\alpha$, there exists a $C^{k, \beta}$-family of (generic) 
submanifolds $M_{t}^{\prime}, 0 \leq t \leq \varepsilon^{\prime} \leq \varepsilon$ (for some $\varepsilon^{\prime}$ ), with boundaries $M_{t}$ such that $M_{0}^{\prime}=M^{\prime}$ and all $C R$-functions on $M_{t}$ extend to be $C R$ on $M_{t}^{\prime}$.

(iii) Each submanifold $M_{t}^{\prime}$ in (ii), $0 \leq t \leq \varepsilon^{\prime}$, can be chosen to be the union of open pieces of radii of the form $A((1-\gamma, 1]), \gamma>0$, of sufficiently small analytic discs $A$ of class $\mathcal{C}^{k, \alpha}$ attached to $M_{t}$.

The proof can be obtained by following the proof of Lemma 6.4 in [Tu96] and using Theorem 1.2 in [Tu93] to show the required regularity for the solutions of Bishop's equation with parameters. The details are left to the reader.

For the next statement, recall that, given two open cones $\Gamma^{\prime} \subset \Gamma \subset \mathbb{R}^{n}, \Gamma^{\prime}$ is said to be finer than $\Gamma$, if the intersection of $\Gamma^{\prime}$ with the unit sphere in $\mathbb{R}^{n}$ is relatively compact in $\Gamma$. The following result is a deformation version of a theorem of Ajrapetian-Henkin [AH81]. We keep the notation from Definition 2.2.

Proposition 4.3. Let $M \subset \mathbb{C}^{N}$ be a generic submanifold through 0 of codimension d. Let $M_{1}, \ldots, M_{d}$ be (generic) submanifolds with boundary $M$ at 0 of class $\mathcal{C}^{k, \alpha}(k \geq 1,0<\alpha<1)$ with $\xi_{j} \in T_{0} M_{j}$ pointing into $M_{j}$ such that $T_{0} \mathbb{C}^{N}=T_{0} M \oplus \mathbb{R} \xi_{1} \oplus \cdots \oplus \mathbb{R} \xi_{d}$. Set $\Gamma:=\mathbb{R}_{+} \xi_{1}+\cdots+\mathbb{R}_{+} \xi_{d}$. Then for any finer cone $\Gamma^{\prime} \subset \Gamma$, the following hold:

(i) All continuous functions on $M \cup \bigcup_{j=1}^{d} M_{j}$ that are $C R$ on each $M_{j}$ extend to be holomorphic in a (standard) wedge $W$ with edge $M$ at 0 in the direction $\Gamma^{\prime}$.

(ii) Moreover, given a $\mathcal{C}^{k, \alpha}$-family of (generic) submanifolds $\left(M_{t}\right)_{0 \leq t \leq \varepsilon}$ with $M_{0}=M, \mathcal{C}^{k, \alpha}$-families $M_{j, t}, 0 \leq t \leq \varepsilon$, of (generic) submanifolds with boundaries $M_{t}$ with $M_{j, 0}=M_{j}, 1 \leq j \leq d$, and given any $0<\beta<\alpha$, there exists a $\mathcal{C}^{k, \beta}$-family of open subsets $U_{t} \subset M_{t}, 0 \leq t \leq \varepsilon^{\prime}<\varepsilon$ (for some $\varepsilon^{\prime}$ ), with $0 \in U_{0}$, and a neighborhood $\Omega$ of 0 in the linear span of $\Gamma$ such that all continuous functions on $M_{t} \cup \bigcup_{j=1}^{d} M_{j, t}$ that are $C R$ on each $M_{j, t}, 1 \leq j \leq d$, extend to be holomorphic in the (standard) wedge $W_{t}:=W\left(U_{t}, \Omega, \Gamma^{\prime}\right)$ for each $0 \leq t \leq \varepsilon^{\prime}$.

(iii) Each wedge $W_{t}$ given in (ii), $0 \leq t \leq \varepsilon^{\prime}$, can be obtained as a union of centers $A(0)$ of sufficiently small analytic discs $A$ of class $\mathcal{C}^{k, \alpha}$ attached to $M_{t} \cup \bigcup_{j=1}^{d} M_{j, t}$.

The proof can be obtained by following the proof of Theorem 4.1 in [Tu96] and using Theorem 1.2 in [Tu93] as before to show the required regularity with respect to the parameters. We omit further details.

Proof of Theorem 2.4. We may assume that $p=0$. Since the generic submanifold $M$ is minimal at 0 , it follows from [Tu88, Tu90] that there exists a (standard) wedge $W=W\left(U_{1}, \Omega_{1}, \Gamma^{1}\right)$ with edge $M$ at 0 (whose base is some neighborhood $U_{1}$ of 0 in $M$ ) such that all CR-functions on $M$ holomorphically extend to $W$. We shall prove that any open neighborhood $U \subset U_{1}$ satisfies the conclusion of Theorem 2.4. For this set $W_{1}:=W\left(U, \Omega_{1}, \Gamma^{1}\right)$ and note that any CR-function on $M$ holomorphically extends to $W_{1}$. Let $M_{0} \subset U$ be an open relatively compact neighborhood of 0 and denote by $d$ the codimension of $M$ in $\mathbb{C}^{N}$. 
By applying Tumanov's theorem again to the open subset $M_{0} \subset M$, we obtain another (standard) wedge $W_{2}$ with edge $M_{0}$ at 0 , where all CR-functions on $M_{0}$ holomorphically extend. We shall need more precise information about the wedge $W_{2}$ that can be obtained from the theory of defects of analytic discs [Tu88] (see also the geometric definition due to BAOUENDI-ROTHSCHILD-TRÉPREAU [BRT94] that has been used in [Tu96]). Here the $\mathcal{C}^{2, \alpha}$-smoothness of $M_{0}$ is required to guarantee the $\mathcal{C}^{1, \alpha}$-smoothness of the conormal bundle to $M_{0}$ needed for Bishop's equation. By the theory mentioned above (see e.g. the proof of Theorem 6.1 in [Tu96]) and the minimality of $M_{0}$ at 0 , there exist $d$ arbitrarily small analytic discs $A_{j}$ of class $\mathcal{C}^{2, \alpha}, 1 \leq j \leq d$, attached to $M_{0}$ such that $A_{j}(1)=0$ and

$$
T_{0} \mathbb{C}^{N}=T_{0} M \oplus \mathbb{R} v_{1} \oplus \cdots \oplus \mathbb{R} v_{d}
$$

where $v_{j}:=\partial_{\nu} A_{j}(1)$ for $1 \leq j \leq d$. Therefore, from Proposition 4.2 (i), for any fixed $0<\beta<\alpha$, we obtain submanifolds $M_{j}, j=1, \ldots, d$, with boundary $M$ at 0 of class $\mathcal{C}^{2, \beta}$ with $T_{0} M_{j}=T_{0} M \oplus \mathbb{R} v_{j}$ and such that all CR-functions on $M$ extend to be $\mathrm{CR}$ on $\cup_{j=1}^{d} M_{j}$. According to Proposition 4.3 (i), the wedge $W_{2}$ can be chosen to have the form $W_{2}:=W\left(U_{2}, \Omega_{2}, \Gamma^{2}\right)$, where $\Gamma^{2} \subset \mathbb{R}_{+} v_{1} \oplus \cdots \oplus \mathbb{R}_{+} v_{d}$ is any finer cone and $U_{2} \subset M_{0}$ is some neighborhood of 0 in $M$.

Choose $(d-1)$ vectors $\xi_{1}, \ldots, \xi_{d-1} \in \Gamma^{1}$ and one vector $\xi_{d} \in \Gamma^{2}$ such that $T_{0} \mathbb{C}^{N}=T_{0} M \oplus \mathbb{R} \xi_{1} \oplus \cdots \oplus \mathbb{R} \xi_{d}$. Applying Proposition 4.3 (i) to these $d$ vectors (with suitably chosen submanifolds with boundary $M$ in their directions), we obtain a new (standard) wedge $W_{3}:=W\left(U_{3}, \Omega_{3}, \Gamma^{3}\right)$ with edge $M$ at $0, U_{3} \subset$ $U_{2}$, where all CR-functions on $M$ holomorphically extend, and such that the direction cone $\Gamma^{3}$ has nonempty intersection with both $\Gamma^{1}$ and $\Gamma^{2}$. Fix a vector $\xi_{0} \in \Gamma^{3} \cap \Gamma^{2}$. We claim that any generalized wedge $\mathcal{W}$ (as in Definition 2.2) with base $U$, directions $\left(\Gamma_{x}\right)_{x \in U}$, such that $\mathcal{W} \subset W_{1} \cup W_{2} \cup W_{3}$ and $\xi_{0} \in \Gamma_{0}$, satisfies the conclusion of Theorem 2.4. The existence of such a generalized wedge $\mathcal{W}$ is obvious from the construction. Note that unless $\xi_{0} \in \Gamma^{1}, \mathcal{W}$ cannot be chosen to be a standard wedge.

For the rest of the proof, we fix $\mathcal{W}$ satisfying the above properties. Then conclusion (i) of Theorem 2.4 follows directly from the construction and the inclusion $\mathcal{W} \subset W_{1} \cup W_{2} \cup W_{3}$. To show (ii), we fix a smooth section $\eta: U \rightarrow T \mathbb{C}^{N}$ with $\eta(x) \in \Gamma_{x}$ for every $x \in U$ and such that $\eta(0)=\xi_{0}$. We choose the section $\eta$ to be $\mathcal{C}^{\infty}$-extendable to a neighborhood of $U$ in $\mathbb{C}^{N}$. Fix also a reflection $\nu: \omega \rightarrow \omega$ with respect to $M$ as in Definition 2.1. Then, by the construction of $\eta$, we have

$$
M_{t}:=\left\{x-t \eta(x): x \in M_{0}\right\} \subset \nu(\mathcal{W} \cap \omega)
$$

for all sufficiently small $t \geq 0$, say $0 \leq t \leq \varepsilon$. Unless $\xi_{0} \in \Gamma^{1}$, we cannot choose $\eta$ to be constant. This is the exactly the reason why, in what follows, we need the deformation parts of Propositions 4.2 and 4.3 that we shall apply to the $\mathcal{C}^{2, \alpha}$ family of generic submanifolds $\left(M_{t}\right)_{0 \leq t \leq \varepsilon}$ given by (4.3). 
From Propositions 4.2 and 4.3 (ii), we conclude the existence of wedges $W_{t}:=$ $W\left(V_{t}, \Omega, \Gamma^{2}\right), V_{t} \subset M_{t}, 0 \leq t \leq \varepsilon^{\prime} \leq \varepsilon$, such that all CR-functions on $M_{t}$ extend holomorphically to $W_{t}$. Here it is very important that all wedges $W_{t}, 0 \leq t \leq \varepsilon^{\prime}$, have the same direction $\Gamma^{2}$ and the same "size" given by $\Omega$. Since $\eta(0)=\xi_{0} \in \Gamma^{2}$, it follows from the construction of $M_{t}$ that there exists $t_{0}>0$ sufficiently small such that $W_{t_{0}}$ contains a neighborhood $U(0)$ of 0 in $\mathbb{C}^{N}$. Since all holomorphic functions in $\nu(\mathcal{W} \cap \omega)$ restrict to CR-functions on $M_{t_{0}}$, it follows that they extend holomorphically to $W_{t_{0}}$ and therefore to $U(0)$. This finishes the proof of (ii) and hence completes the proof of Theorem 2.4.

\section{Meromorphic extension of CR-functions; proof of Theorem 2.6}

In this section, we prove Theorem 2.6. We follow the proof of [CPS99] in certain parts and deviate from it at other places, where another approach is required. We also apply different methods to avoid removing singularities.

The first step is to use a real-analytic reflection $\nu$ with respect to $M$ (as in Definition 2.1) which is antiholomorphic only in certain coordinates. The following standard lemma is implicitly contained in [BJT85]. For the reader's convenience we give a short proof. As in $\S 3$, for a point $q \in M$, we denote by $T_{q}^{c} M$ the complex tangent space to $M$ at $q$.

Lemma 5.1. Let $M \subset \mathbb{C}^{N}$ be a generic real-analytic submanifold of through a point $p \in M$ and $L$ a complex linear subspace of $\mathbb{C}^{N}$ such that

$$
T_{p}^{c} M \oplus L=\mathbb{C}^{N}
$$

Then there exists a connected neighborhood $\omega$ of $p$ in $\mathbb{C}^{N}$ and a real-analytic reflection $\nu: \omega \rightarrow \omega$ with respect to $M \cap \omega$ which sends each affine subspace $q+L, q \in M \cap \omega$, antiholomorphically into itself. For any given $\omega$, the reflection $\nu$ with the above property is unique.

Proof. Let $d$ be the codimension of $M$ in $\mathbb{C}^{N}$ and $n$ the (complex) dimension of $T_{p}^{c} M$ so that one has, in particular, $N=n+d$. We choose complex-linear coordinates $z=(\chi, \tau) \in \mathbb{C}^{n} \times \mathbb{C}^{d}$ vanishing at $p$ such that $L=\{\chi=0\}$. Then, near 0 , there exists a real-analytic parametrization of $M$ given by

$$
\mathbb{C}^{n} \times \mathbb{R}^{d} \ni(\chi, s) \mapsto(\chi, \psi(\chi, \bar{\chi}, s)) \in M
$$

whose complexification with respect to $s$ is denoted by $\Psi(\chi, \bar{\chi}, \tau)$, where $(\chi, \tau) \in$ $\mathbb{C}^{n} \times \mathbb{C}^{d}$. It is easy to see that $\Psi$ is a local diffeomorphism of $\mathbb{C}^{N}$ near 0 which is holomorphic in $\tau$ and sends each subspace $q+L$ into itself. It remains to define $\widetilde{\nu}(\chi, \tau):=(\chi, \bar{\tau})$ and to set $\nu:=\Psi \circ \widetilde{\nu} \circ \Psi^{-1}$. If $\nu$ is defined in a neighborhood $U$ of $p$ in $\mathbb{C}^{N}$, by setting $\omega:=U \cap \nu(U)$, it is then easy to check that $\nu$ satisfies the desired properties. (If $U \cap \nu(U)$ is not connected, we take for $\omega$ the component of $U \cap \nu(U)$ containing $p$.) The uniqueness of $\nu$ is due to the facts that $\nu$ is antiholomorphic on $q+L$ and, by (5.1), $M \cap(q+L)$ is generic in $q+L$ near $q$, for $q \in M$ near $p$.

We shall use the following notion: 
Definition 5.2. Let $L \subset \mathbb{C}^{N}$ be a complex vector subspace. We say that a function $h$ defined on an open subset of $\mathbb{C}^{N}$ is holomorphic along $L$ if, for each fixed $q \in \mathbb{C}^{N}$, the restriction of $h$ to the affine subspace $q+L \subset \mathbb{C}^{N}$ is holomorphic on its domain of definition.

We then have the following obvious extension property:

Lemma 5.3. Let $M, p$ and $L$ be as in Lemma 5.1 and $\nu: \omega \rightarrow \omega$ be the corresponding real-analytic reflection. Let $g: M \rightarrow \mathbb{C}$ be a continuous function that extends holomorphically to a generalized wedge $\mathcal{W} \subset \omega$ with edge $M$ at $p$ (and continuous up to $M)$. Then the conjugate function $M \ni z \mapsto \overline{g(z)} \in \mathbb{C}$ extends to a real-analytic function on $\nu(\mathcal{W})$, holomorphic along $L$, and continuous up to $M$. Moreover, for any continuous function on $M$, such an extension to $\nu(\mathcal{W})$, if it exists, is always unique.

Proof. The required extension is given by $\nu(W) \ni z \mapsto \overline{g(\nu(z))}$. The uniqueness follows from the boundary uniqueness theorem applied to the intersections of $M$ with the affine subspaces $q+L \subset \mathbb{C}^{N}$ for $q \in M$ near $p$.

Our proof of Theorem 2.6 will use the following result of SHIfFMAN [Sh91]:

Theorem 5.4. Let $U \subset \mathbb{C}^{n}$ and $V \subset \widetilde{V} \subset \mathbb{C}^{m}$ be domains and $h$ a meromorphic function on $U \times V$. Assume that, for each fixed $\chi \in U$, either $h(\chi, \cdot)$ extends meromorphically to $\widetilde{V}$ or $\{\chi\} \times V$ is contained in the pole set of $h$. Then $h$ extends meromorphically to $U \times \widetilde{V}$.

Proof of Theorem 2.6. To prove Theorem 2.6, it is sufficient to show the local statement that, for each point $p \in M, v(z, \overline{g(z)})$ does not vanish on a dense subset of a neighborhood of $p$ in $M$ and the quotient (2.1) that we denote by $h$ extends meromorphically to a neighborhood of $p$ in $\mathbb{C}^{N}$. Let $\Omega \subset M$ be the set of all points $p \in M$ with the above property. Clearly $\Omega$ is open in $M$.

We first show that $\Omega$ is nonempty. Indeed, by the assumption, there exists a point $p \in M$ such that $h$ is defined and CR in a neighborhood of $p$ in $M$. By the minimality of $M$ at $p$, both $g$ and $h$ extend holomorphically to a (standard) wedge $W$ with edge $M$ at $p$. Choose any $L$ satisfying the assumptions of Lemma 5.1 and let $\nu: \omega \rightarrow \omega$ be the reflection given by that lemma. We may assume that $W \subset \omega$. Then by Lemma 5.3, $\overline{g(z)}$ and therefore $h(z)$ extend to real-analytic functions on $\nu(W) \cap U(p)$, holomorphic along $L$ and continuous up to $M$, where $U(p)$ is some neighborhood of $p$ in $\mathbb{C}^{N}$. Finally, it follows from the edge-of-the-wedge theorem (see e.g. [BER99, Theorem 7.6.1] and the references there) and from Proposition 1.7.5 in [BER99] that $h$ extends holomorphically to a neighborhood of $p$ in $\mathbb{C}^{N}$. Hence $\Omega$ is not empty. (The above holomorphic extension of $h$ can be also obtained as a special case of the argument below showing meromorphic extension instead of the holomorphic one.)

We need to prove that $\Omega=M$. We claim that it is sufficient to show that, if $C \subset M$ is any real-analytic curve with $T_{x} C \not \subset T_{x}^{c} M$ for each $x \in C$, then 
$C \cap \Omega \neq \emptyset$ implies $C \subset \Omega$. Indeed, any two points of $M$ can be connected by a chain of curves with the mentioned transversality property.

We now fix such a curve $C \subset M$ with $C \cap \Omega \neq \emptyset$ and suppose, by contradiction, that $C \not \subset \Omega$. Then, from the fact that $\Omega$ is open, we see that there exists a point $p \in C \backslash \Omega$ that is contained in the closure of $C \cap \Omega$. We choose local holomorphic coordinates in $\mathbb{C}^{N}$ vanishing at $p$ such that $C$ becomes a real line. Because of the property $T_{p} C \not \subset T_{p}^{c} M$, we can choose a complex vector subspace $L \subset \mathbb{C}^{N}$ (possibly different from the previous one) passing through $C$ and satisfying (5.1). By a linear change of coordinates $(\chi, \tau) \in \mathbb{C}^{n} \times \mathbb{C}^{d}$, we may assume that $L=$ $\{\chi=0\}$ and $M$ is locally given by $\operatorname{Im} \tau=\varphi(\chi, \bar{\chi}, \operatorname{Re} \tau)$ for some real-analytic function $\varphi$ defined near $0 \in \mathbb{R}^{2 n+d}$. (Here the integers $d$ and $n$ are as in the proof of Lemma 5.1.) We further define a neighborhood basis $\left(U_{k}\right)_{k \geq 1}$ of $p$ in $M$ by

$$
U_{k}:=\left\{(\chi, \tau) \in M:|\chi|<\varepsilon_{k},|\operatorname{Re} \tau|<1 / k\right\}
$$

where $0<\varepsilon_{k}<1 / k$ is chosen such that $(q+L) \cap U_{k} \cap \Omega \neq \emptyset$ for every $q \in U_{k}$. Such a choice of $\varepsilon_{k}$ is always possible because $C \subset L, p$ is contained in the closure of $C \cap \Omega$ and $\Omega$ is open in $M$.

Let $\nu: \omega \rightarrow \omega$ be the real-analytic reflection given by Lemma 5.1 (for the new subspace $L$ ). By shrinking $M$ around $p$, we may assume that $M \subset \omega$. We finally set $\Pi:=\{\chi=0, \operatorname{Re} \tau=0\}$ so that $T_{p} M \oplus \Pi=\mathbb{C}^{N}$. By Theorem 2.4, there exists a generalized wedge $\mathcal{W} \subset \omega$ with edge $M$ at $p$, whose base is $U_{k}$ for some $k$, satisfying properties (i) and (ii) of that theorem. By (i), the CR-function $g$ admits a holomorphic extension to $\mathcal{W}$ denoted by $\widetilde{g}$.

By Lemma 5.3, $\overline{g(z)}$ extends to a real-analytic function on $\nu(\mathcal{W})$ that is holomorphic along $L$. By composing with holomorphic functions $u$ and $v$, we conclude that the functions $u(z, \overline{g(z)})$ and $v(z, \overline{g(z)})$ extend to real-analytic functions $\widetilde{u}(z)$ and $\widetilde{v}(z)$ respectively in a neighborhood of $M$ in $\nu(\mathcal{W})$, holomorphic along $L$ and continuous up to $M$. By shrinking $\omega$ and then $\mathcal{W}$, we may assume that this extension takes place in $\nu(\mathcal{W}) \subset \omega$. The assumption $C \cap \Omega \neq \emptyset$ implies that the extension $\widetilde{v}$ cannot vanish identically and therefore vanishes on a closed proper real-analytic subset $\Sigma \subset \nu(\mathcal{W})$. Moreover, the uniqueness part of Lemma 5.3 implies that $v(z, \overline{g(z)})$ does not vanish on a dense subset of the base $U_{k}$. It follows then that the quotient $h$ extends to a real-analytic function $\widetilde{h}:=\widetilde{u} / \widetilde{v}$ on $\nu(\mathcal{W}) \backslash \Sigma$ that is holomorphic along $L$. Hence, for each $q \in U_{k}$ either $(q+L) \cap \nu(\mathcal{W}) \subset\{z \in \nu(\mathcal{W}): \widetilde{v}(z)=0\}$, or $\left.\widetilde{h}\right|_{q+L}$ is meromorphic.

We claim that $\widetilde{h}$ extends to a meromorphic function on $\nu(\mathcal{W})$. Since $\widetilde{h}$ is already defined on a dense open subset of $\nu(\mathcal{W})$, it is sufficient to show that $\widetilde{h}$ extends meromorphically to a neighborhood of any given point $z_{0}=\left(\chi_{0}, \tau_{0}\right) \in$ $\nu(\mathcal{W})$. By the construction and by Definition 2.2, for any $z_{0} \in \nu(\mathcal{W})$, there exists a point $q_{0} \in\left(z_{0}+L\right) \cap U_{k} \cap \Omega$. By the definition of the set $\Omega \subset M, h$ extends to a meromorphic function $\widetilde{h}^{\prime}$ in a neighborhood $G$ of $q_{0}$ in $\mathbb{C}^{N}$. On the 
dense subset of $U_{k}$, where $h$ is defined, we have the identity

$$
v(z, \overline{g(z)}) h(z) \equiv u(z, \overline{g(z)}) .
$$

Without loss of generality, $G \cap \nu(\mathcal{W})$ is connected. Then, by the uniqueness part of Lemma 5.3, (5.3) implies $\widetilde{v} \widetilde{h}^{\prime}=\widetilde{u}$ on $G \cap \nu(\mathcal{W})$. In particular, we obtain $\widetilde{h}^{\prime}=\widetilde{h}$ on $G \cap(\nu(\mathcal{W}) \backslash \Sigma)$. Now it is clear that there exist domains $U \subset \mathbb{C}^{n}$ and $V \subset \widetilde{V} \subset \mathbb{C}^{d}$ such that $q_{0} \in U \times V \subset G$ and $z_{0} \subset U \times \widetilde{V} \subset G \cup \nu(\mathcal{W})$. We may thus apply Theorem 5.4 to conclude that $\widetilde{h}$ extends meromorphically to $U \times \widetilde{V}$ and, in particular, to a neighborhood of $z_{0}$ as required.

Since $z_{0} \in \nu(\mathcal{W})$ was chosen arbitrarily, we conclude that $\widetilde{h}$ extends meromorphically to $\nu(\mathcal{W})$. Therefore Corollary 2.5 yields the required meromorphic extension of $\widetilde{h}$ to a neighborhood of $p$ and hence that of $h$. This contradicts our assumption that $p \notin \Omega$ and thus proves that $\Omega=M$. The proof of Theorem 2.6 is complete.

\section{Analyticity of CR-mappings; proof of Theorem 1.1}

Let $M \subset \mathbb{C}^{N}$ be a generic real-analytic submanifold through a point $p \in \mathbb{C}^{N}$. Following [CPS99], we denote by $\mathcal{A}_{p}(M)$ the ring of germs at $p$ of $\mathcal{C}^{\infty}$-smooth functions $\varphi$ on $M$ that can be written near $p$ in the form

$$
\varphi(z)=\Phi(z, \overline{g(z)}), z \in M,
$$

where $g$ is a $\mathcal{C}^{\infty}$-smooth CR-function in a neighborhood of $p$ in $\mathbb{C}^{N}$ with values in $\mathbb{C}^{k}$ for some $k$, and $\Phi$ is a holomorphic function near $(p, \overline{g(p)})$. Note that the ring of germs at $p$ of real-analytic functions on $M$ is contained in $\mathcal{A}_{p}(M)$. In what follows, we write $\mathcal{O}_{p}(M)$ for the subring of $\mathcal{A}_{p}(M)$ consisting of all restrictions of germs (at $p$ ) of holomorphic functions in $\mathbb{C}^{N}$. Observe that, if the submanifold is furthermore assumed to be minimal at $p$, it follows from TuMANOV's theorem and Lemma 5.3 that the ring $\mathcal{A}_{p}(M)$ is an integral domain. We further need the following lemma.

Lemma 6.1. Let $M \subset \mathbb{C}^{N}$ be a generic real-analytic minimal submanifold through a point $p \in \mathbb{C}^{N}, \varphi$ a germ in $\mathcal{A}_{p}(M)$ and $L$ a real-analytic $(0,1)$ vector field on $M$ defined near $p$. Then $L \varphi$ is also in $\mathcal{A}_{p}(M)$.

Proof. We first consider the case when $\varphi(z)=\overline{g(z)}$, where $g$ is a CR-function in a neighborhood of $p$ in $M$. Write $L=\sum_{j=1}^{N} a_{j}(z, \bar{z})\left(\partial / \partial \overline{z_{j}}\right)$ with $a_{j}(z, \bar{z})$ real-analytic near $p$. By TuMANOV's theorem, $g$ extends holomorphically to a wedge $W$ with edge $M$ at $p$. Moreover, this extension that we call $\widetilde{g}$ is smooth up to the edge (see e.g. [BER99, Theorem 7.5.1]). The partial derivatives $\partial \widetilde{g} / \partial z_{j}$, $j=1, \ldots, N$, are holomorphic in $W$ and have boundary values on $M$ that are smooth CR-functions. Denote by $\widetilde{L}=\sum_{j=1}^{N} \widetilde{a}_{j}(z, \bar{z})\left(\partial / \partial \bar{z}_{j}\right)$ any real-analytic extension of $L$ as a $(0,1)$ vector field in a neighborhood of $p$ in $\mathbb{C}^{N}$. Then $L \varphi$ is 
the boundary value of the function

$$
\widetilde{L} \overline{\widetilde{g}}=\sum_{j=1}^{N} \widetilde{a}_{j}(z, \bar{z}) \overline{\partial \widetilde{g} / \partial z_{j}},
$$

and therefore can be written as a holomorphic function in $z, \bar{z}$ and the boundary values of $\overline{\partial g / \partial z_{1}}, \ldots, \overline{\partial g / \partial z_{N}}$. This shows that $L \varphi \in \mathcal{A}_{p}(M)$.

To prove the statement for an arbitrary $\varphi \in \mathcal{A}_{p}(M)$, write $\varphi(z)=\Phi(z, \overline{g(z)})$ as in (6.1), $\Phi=\Phi(\zeta, z)$, and apply the chain rule to conclude

$$
(L \varphi)(z)=\sum_{j} \Phi_{\zeta_{j}}(z, \overline{g(z)})\left(L \overline{g_{j}}\right)(z) .
$$

Since $L \overline{g_{j}} \in \mathcal{A}_{p}(M)$ by the first part of the proof, we have $L \varphi \in \mathcal{A}_{p}(M)$ as required.

Proposition 6.2. Let $M \subset \mathbb{C}^{N}$ be a generic real-analytic submanifold through a point $p \in \mathbb{C}^{N}$ and $h$ a germ at $p$ of a $\mathcal{C}^{\infty}$-smooth $C R$-function on $M$. Assume that $M$ is minimal at $p$ and that $h$ satisfies a nontrivial polynomial identity with coefficients in $\mathcal{A}_{p}(M)$. Then $h$ also satisfies a nontrivial polynomial identity with coefficients in $\mathcal{O}_{p}(M)$.

Proof. Choose a nontrivial polynomial $P(T)=\sum_{j=0}^{r} P_{j} T^{j}, P_{j} \in \mathcal{A}_{p}(M)$, of minimal degree $r$ satisfying for $z \in M$ close to $p$,

$$
\sum_{j=0}^{r} P_{j}(z)(h(z))^{j} \equiv 0, \text { and } P_{r}(z) \not \equiv 0 .
$$

For $j=1, \ldots, r$, we may write $P_{j}(z)=\Phi_{j}(z, \overline{g(z)})$, where $g: M \rightarrow \mathbb{C}^{k}$ is a CR-map near $p$ and each $\Phi_{j}$ is holomorphic near $(p, \overline{g(p)})$. Therefore, we may rewrite $(6.2)$ as

$$
\sum_{j=0}^{r} \Phi_{j}(z, \overline{g(z)})(h(z))^{j} \equiv 0
$$

for $z \in M$ close to $p$.

We claim that the quotients

$$
\Theta_{j}(z):=\frac{\Phi_{j}(z, \overline{g(z)})}{\Phi_{r}(z, \overline{g(z)})}, \quad j=0, \ldots, r-1,
$$

are $\mathrm{CR}$ on their domains of definition. Indeed, otherwise we could choose one real-analytic $(0,1)$ vector field $L$ for $M$ near $p$ and some $1 \leq j_{0}<r$ such that $L \Theta_{j_{0}} \not \equiv 0$. Then we could divide both sides of (6.3) by the nontrivial function $\Phi_{r}(\overline{g(z)}, z)$ and apply $L$ to obtain, in view of Lemma 6.1, another nontrivial polynomial $\widetilde{P}(T)$ of degree strictly less than $r$ with coefficients in $\mathcal{A}_{p}(M)$ satisfying $\widetilde{P}(h) \equiv 0$. This would contradict the choice of $P$ and hence the claim follows. 
Now, by Theorem 2.6, each quotient $\Theta_{j}(z), j=0, \ldots, r-1$, extends meromorphically to a neighborhood of $p$ in $\mathbb{C}^{N}$. By writing each meromorphic extension as a quotient of two holomorphic functions near $p$, we obtain the required conclusion in view of (6.3). This completes the proof of Proposition 6.2.

As a consequence of theorems of MaLgrange (see e.g. [BHR96]) and Tomassini [To66], Proposition 6.2 implies the following:

Corollary 6.3. Let $M \subset \mathbb{C}^{N}$ be a generic real-analytic submanifold through a point $p \in \mathbb{C}^{N}$ and $h$ be a germ at $p$ of a $\mathcal{C}^{\infty}$-smooth $C R$-function on $M$. Assume that $M$ is minimal at $p$ and that $h$ satisfies a nontrivial polynomial identity with coefficients in $\mathcal{A}_{p}(M)$. Then $h$ extends holomorphically to a neighborhood of $p$ in $\mathbb{C}^{N}$.

Finally, we mention the following well-known fact (see e.g. [BER99, Theorem 5.3.9]) which will be needed for the proof of Theorem 1.1:

Proposition 6.4. Let $A \subset \mathbb{C}_{z}^{m} \times \mathbb{C}_{w}^{l}$ be a complex-analytic subset through 0 such that $A \cap\left(\{0\} \times \mathbb{C}^{l}\right)=\{0\}$. Then for $j=1, \ldots$, l, there exist Weierstra $\beta$ polynomials

$$
Q_{j}\left(z, w_{j}\right)=w_{j}^{r_{j}}+\sum_{k=0}^{r_{j}-1} Q_{j k}(z) w_{j}^{k}, \quad w=\left(w_{1}, \ldots, w_{l}\right),
$$

such that for every $(z, w) \in A$ near $0, Q_{j}\left(z, w_{j}\right)=0$ holds for all $j$.

Proof of Theorem 1.1. Let $M, M^{\prime}, f$ and $p \in M$ satisfy the assumptions of Theorem 1.1. Without loss of generality, we may assume $M$ to be generic. Denote by $d^{\prime}$ the codimension of the submanifold $M^{\prime}$ in $\mathbb{C}^{N^{\prime}}$, by $n$ the (complex) dimension of $T_{p}^{c} M$ (cf. $\S 3$ ). Choose a set of real-analytic defining functions $\rho^{\prime}\left(z^{\prime}, \overline{z^{\prime}}\right)=\left(\rho_{1}^{\prime}\left(z^{\prime}, \overline{z^{\prime}}\right), \ldots, \rho_{d^{\prime}}^{\prime}\left(z^{\prime}, \overline{z^{\prime}}\right)\right)$ for $M^{\prime}$ defined in some neighborhood $U^{\prime}$ of $p^{\prime}$ in $\mathbb{C}^{N^{\prime}}$. Shrinking $M$ if necessary around $p$, we may assume that, for every $z \in M$, we can fix a holomorphic parametrization $\gamma_{z}:\left(\mathbb{C}^{n}, 0\right) \rightarrow\left(Q_{z}, z\right)$ depending real-analytically on $z$. Recall here that $Q_{z}$ denotes the Segre variety of $z$ associated to the generic submanifold $M$, as defined in (1.1). We may also assume that $f(M) \subset U^{\prime} \cap M^{\prime}$.

Recall that for all points $z \in M$, the Taylor series of $f$ at $z$ extends to a formal (holomorphic) power series mapping $F_{z}:\left(\mathbb{C}^{N}, z\right) \rightarrow\left(\mathbb{C}^{N^{\prime}}, f(z)\right)$. In view of (1.2) (see also $\S 3$ ), a point $w^{\prime} \in U^{\prime}$ belongs to $r_{z}$ if and only if the following formal power series identity

$$
\rho^{\prime}\left(F_{z}\left(\gamma_{z}(t)\right), \overline{w^{\prime}}\right) \equiv 0
$$

holds in the ring $\mathbb{C}[[t]], t=\left(t_{1}, \ldots, t_{n}\right)$. Differentiating (6.4) with respect to $t$ and evaluating at $t=0$ yields that $w^{\prime} \in r_{z}$ if and only if, for all $k \in \mathbb{N}$,

$$
0 \equiv j_{t}^{k}\left(\rho^{\prime}\left(F_{z}\left(\gamma_{z}(t)\right), \overline{w^{\prime}}\right)\right)(0):=\left(\left.\partial_{t}^{\nu}\left(\rho^{\prime}\left(F_{z}\left(\gamma_{z}(t)\right), \overline{w^{\prime}}\right)\right)\right|_{t=0}\right)_{|\nu| \leq k} .
$$


It follows from the chain rule that there exists a universal polynomial mapping $P_{k}$ such that

$$
j_{t}^{k}\left(\rho^{\prime}\left(F_{z}\left(\gamma_{z}(t)\right), \overline{w^{\prime}}\right)\right)(0) \equiv P^{k}\left(\left(j_{z^{\prime}}^{k} \rho^{\prime}\right)\left(f(z), \overline{w^{\prime}}\right),\left(j_{z}^{k} f\right)(z),\left(j_{t}^{k} \gamma_{z}\right)(0)\right) .
$$

Here as in (6.5), we have used standard notations relative to jet spaces i.e.

$$
\begin{aligned}
\left(j_{z^{\prime}}^{k} \rho^{\prime}\right)\left(z^{\prime}, \overline{z^{\prime}}\right) & :=\left(\left(\partial_{z^{\prime}}^{\alpha} \rho^{\prime}\right)\left(z^{\prime}, \overline{z^{\prime}}\right)\right)_{|\alpha| \leq k}, \\
\left(j_{t}^{k} \gamma_{z}\right)(t) & :=\left(\left(\partial_{t}^{\beta} \gamma_{z}\right)(t)\right)_{|\beta| \leq k}, \\
\left(j_{z}^{k} f\right)(z) & :=\left(\partial_{z}^{\alpha} f(z)\right)_{|\alpha| \leq k} .
\end{aligned}
$$

The last derivatives are understood as boundary values on $M$ of the partial derivatives of the holomorphic extension of $f$ to a wedge with edge $M$ (such an extension is a consequence of the minimality of $M$ at $p$ ). We write the mapping in the right-hand side of (6.6) as

$$
\Psi_{k}\left(\bar{z}, g_{k}(z), \overline{w^{\prime}}\right)
$$

where $g_{k}(z):=\left(z, j_{z}^{k} f(z)\right)$ is $\mathrm{CR}$ on $M$ and $\Psi_{k}$ is holomorphic in a neighborhood of $\left(\bar{p}, p, j_{z}^{k} f(p), \overline{f(p)}\right)$. Note also that, for all $z \in M$, the map $w^{\prime} \mapsto$ $\Psi_{k}\left(\bar{z}, g_{k}(z), \overline{w^{\prime}}\right)$ is defined and antiholomorphic in $U^{\prime}$. By the definition of $r_{z}$, $z \in M$, we have

$$
r_{z}=\left\{w^{\prime} \in U^{\prime}: \Phi_{k}\left(z, \overline{g_{k}(z)}, w^{\prime}\right)=0 \text { for all } k \in \mathbb{N}\right\},
$$

where $\Phi_{k}$ is the conjugate of $\Psi_{k}$.

By the Noetherian property, there exist a neighborhood $\widetilde{U}^{\prime} \subset U^{\prime}$ of $p^{\prime}$ and a positive integer $\kappa$ such that $w^{\prime} \in r_{p} \cap \widetilde{U}^{\prime}$ if and only if $\Phi_{\kappa}\left(p, \overline{g_{\kappa}(p)}, w^{\prime}\right)=0$. If $J_{p}^{\kappa}\left(\mathbb{C}^{N}, \mathbb{C}^{N^{\prime}}\right)$ denotes the jet space at $p$ of order $\kappa$ of holomorphic mappings from $\mathbb{C}^{N}$ to $\mathbb{C}^{N^{\prime}}$ equipped with coordinates $\Lambda=\left(\Lambda_{\beta}\right)_{|\beta| \leq \kappa}, \beta \in \mathbb{N}^{N}, \Lambda_{\beta} \in \mathbb{C}^{N^{\prime}}$, we define a complex-analytic subset $A \subset \mathbb{C}_{z}^{N} \times \mathbb{C}_{w}^{N} \times J_{p}^{\kappa}\left(\mathbb{C}^{N}, \mathbb{C}^{N^{\prime}}\right) \times \mathbb{C}_{w^{\prime}}^{N^{\prime}}$ in a neighborhood of the point $J_{1}:=\left(p, \bar{p}, \overline{j_{z}^{\kappa} f(p)}, f(p)\right)$ by setting

$$
A:=\left\{\left(z, w, \Lambda, w^{\prime}\right): \Phi_{\kappa}\left(z,(w, \Lambda), w^{\prime}\right)=0\right\} .
$$

Then, for $z \in M$ and $w^{\prime} \in U^{\prime}$, it follows from (6.8) that if $w^{\prime} \in r_{z}$, then $\left(z, \bar{z}, \overline{j_{z}^{\kappa} f(z)}, w^{\prime}\right) \in A$. Since, by assumption, $p^{\prime}=f(p)$ is isolated in $r_{p}$, we conclude that $A \cap\left(\left\{\left(p, \bar{p}, \overline{j_{z}^{\kappa} f(p)}\right)\right\} \times \widetilde{U}^{\prime}\right)=\left\{J_{1}\right\}$. Hence, by Proposition 6.2, we obtain Weierstraß polynomials $Q_{j}\left(z, w, \Lambda ; w_{j}^{\prime}\right)$ in $w_{j}^{\prime}$ for $j=1, \ldots, N^{\prime}, w^{\prime}=$ $\left(w_{1}^{\prime}, \ldots, w_{N^{\prime}}^{\prime}\right)$, defined near $J_{1}$ such that, if $\left(z, w, \Lambda, w^{\prime}\right) \in A$ is close enough to $J_{1}$, then $Q_{j}\left(z, w, \Lambda ; w_{j}^{\prime}\right)=0$ for all $j$. Since, for $z \in M$, one always has $f(z) \in r_{z}$, it follows that $\left(z, \bar{z}, \overline{j_{z}^{\kappa} f(z)}, f(z)\right) \in A$. As a consequence, we have

$$
Q_{j}\left(z, \bar{z}, \overline{j_{z}^{\kappa} f(z)} ; f_{j}(z)\right)=0
$$

for $j=1, \ldots, N^{\prime}$, and $z \in M$ close to $p$. From (6.9), we conclude that each component of $f$ satisfies near $p$ a nontrivial polynomial identity with coefficients in $\mathcal{A}_{p}(M)$ (as defined above). To conclude that $f$ extends holomorphically to a neighborhood of $p$ in $\mathbb{C}^{N}$, it suffices to apply Corollary 6.3. 
Proof of Corollary 1.3. The proof follows from Theorem 1.1 and the fact that, under the assumptions of Corollary 1.3, the point $f(p)$ is isolated in $r_{p}$. The latter fact can be proved by following the techniques of [BR88, Me95]. We leave the details to the reader.

We conclude by giving, as mentioned in the introduction, an example of a situation of Theorem 1.1 which is not covered by Corollary 1.3.

Example 6.5. Consider the unit spheres $M_{0}:=\partial \mathbb{B}^{2} \subset \mathbb{C}^{2}$ and $M^{\prime}:=\partial \mathbb{B}^{3} \subset \mathbb{C}^{3}$ and the map $f: M_{0} \times \mathbb{C} \rightarrow M^{\prime}$ given by $f(z, w, v):=\left(z^{2}, \sqrt{2} z w, w^{2}\right)$. It is easy to check that $f$ is totally degenerate but $p^{\prime}=(1,0,0)$ is isolated in $r_{p}$ with $p=(1,0,0)$.

\section{Acknowledgements}

Part of this work was done while the authors were visiting the University of Padova. They would like to thank this institution for its hospitality. They are grateful to G. ZAMPIERI for many helpful discussions about analytic discs and to M.S. BAouendi, P. EBenfelt and L.P. Rothschild for numerous suggestions and remarks. The third author would also like to thank A. BogGess for calling his attention to Example 4.1.

\section{References}

[AH81] Ajrapetyan, R. A., Henkin, G.M., Analytic continuation of CR-functions through the "edge of the wedge," Soviet Math. Dokl. 24, 129-132 (1981); translation from Dokl. Akad. Nauk SSSR 259 (1981), no. 4, 777-781.

[BBR88] Baouendi, M.S., Bell, S. R., Rothschild, L. P., Mappings of three-dimensional CR manifolds and their holomorphic extension, Duke Math. J. 56 (1988), no. 3, 503530.

[BER99] Baouendi, M.S., Ebenfelt, P., and Rothschild, L. P., Real submanifolds in complex space and their mappings, Princeton Mathematical Series, 47. Princeton University Press, Princeton, NJ, 1999.

[BER00] Baouendi, M.S., Ebenfelt, P., and Rothschild, L. P., Convergence and finite determination of formal CR mappings. J. Amer. Math. Soc. 13 (2000), no. 4, 697-723.

[BHR95] Baouendi, M.S., Huang, X., Rothschild, L. P., Nonvanishing of the differential of holomorphic mappings at boundary points, Math. Res. Lett. 2 (1995), no. 6, 737750 .

[BHR96] Baouendi, M.S., Huang, X., and Rothschild, L. P., Regularity of CR mappings between algebraic hypersurfaces, Invent. Math., 125 (1996), no. 1, 13-36.

[BJT85] Baouendi, M.S., Jacobowitz, H., Treves, F., On the analyticity of CR mappings, Ann. of Math. (2) 122 (1985), 365-400.

[BR88] Baouendi M.S., Rothschild L.P., Germs of CR maps between real-analytic hypersurfaces, Invent. Math. 93 (1988), no. 3, 481-500.

[BR90a] Baouendi, M.S., Rothschild, L.P., Cauchy-Riemann functions on manifolds of higher codimension in complex space, Invent. Math. 101 (1990), no. 1, 45-56.

[BR90b] Baouendi, M.S., Rothschild, L. P., Geometric properties of mappings between hypersurfaces in complex space, J. Differential Geom. 31 (1990), no. 2, 473-499.

[BRT94] Baouendi, M.S., Rothschild, L.P., Trépreau, J.-M., On the geometry of analytic discs attached to real manifolds, J. Differential Geom. 39 (1994), no. 2, 379-405. 
[BT84] Baouendi, M.S., Treves, F., About the holomorphic extension of CR functions on real hypersurfaces in complex space, Duke Math. J. 51 (1984), no. 1, 77-107.

[BP88] Bedford, E., Pinchuk, S., Analytic continuation of biholomorphic maps, Michigan Math. J. 35 (1988), no. 3, 405-408.

[BGN98] Boggess, A., Glenn, L.A., Nagel, A., Model rigid CR submanifolds of CR dimension 1, Pacific J. Math. 184 (1998), no. 1, 43-74.

[CKS84] Cima, J. A., Krantz, S. G., Suffridge, T. J., A reflection principle for proper holomorphic mappings of strongly pseudoconvex domains and applications, Math. Z. 186 (1984), no. 1, 1-8.

[CPS99] Coupet, B., Pinchuk, S., Sukhov, A., Analyticité des applications CR, C. R. Acad. Sci. Paris Sér. I Math. 329 (1999), no. 6, 489-494.

[CPS00] Coupet, B., Pinchuk, S., Sukhov, A., On partial analyticity of CR mappings, Math. Z. 235 (2000), no. 3, 541-557.

[DF88] Diederich K., Fornæss J.E., Proper holomorphic mappings between real analytic pseudoconvex domains in $\mathbb{C}^{n}$, Math. Ann. 282 (1988), no. 4, 681-700.

[DFY94] Diederich, K., Fornæss, J.E., Ye, Z., Biholomorphisms in dimension 2, J. Geom. Anal. 4 (1994), no. 4, 539-552.

[DP95] Diederich K., Pinchuk S., Proper holomorphic maps in dimension two extend, Indiana Univ. Math. J. 44 (1995), no. 4, 1089-1126.

[DW80] Diederich K., Webster S., A reflection principle for degenerate real hypersurfaces, Duke Math. J. 47 (1980), no. 4, 835-843.

[E00] Ebenfelt, P., On the analyticity of CR mappings between nonminimal hypersurfaces, to appear in Math. Ann., http://arxiv.org/abs/math.CV/0009010

[Fa90] Faran, J., A reflection principle for proper holomorphic mappings and geometric invariants, Math. Z. 203 (1990), no. 3, 363-377.

[Fo89] Forstnerič F., Extending proper holomorphic mappings of positive codimension, Invent. Math. 95 (1989), no. 1, 31-61.

[Ha83] Han, C.-K., Analyticity of CR equivalences between some real hypersurfaces with degenerate Levi forms, Invent. Math. 73 (1983), no. 1, 51-69.

[Ha97] Han C.-K., Complete differential system for the mappings of CR manifolds of nondegenerate Levi forms, Math. Ann. 309 (1997), no. 3, 401-409.

[Hu94] Huang, X., On the mapping problem for algebraic real hypersurfaces in the complex spaces of different dimensions, Ann. Inst. Fourier 44 (1994), no. 2, 433-463.

[Hu96] Huang, X., Schwarz reflection principle in complex spaces of dimension two, Comm. Partial Differential Equations 21 (1996), no. 11-12, 1781-1829.

[HP96] Huang, X., Pan, Y., Proper holomorphic mappings between real analytic domains in $\mathbb{C}^{n}$, Duke Math. J. 82 (1996), no. 2, 437-446.

[I92] Ivashkovich, S. M., The Hartogs-type extension theorem for meromorphic maps into compact Kähler manifolds, Invent. Math. 109 (1992), no. 1, 47-54.

[La01] Lamel, B., A reflection principle for real-analytic submanifolds of complex spaces, J. Geom. Anal. 11 (2001), no. 4, 625-631.

[Le56] Lewy, H., On the local character of the solutions of an atypical linear differential equation in three variables and a related theorem for regular functions of two complex variables, Ann. of Math. (2) 64 (1956), 514-522.

[Le77] Lewy, H., On the boundary behaviour of holomorphic mapping,. Contrib. Centro Linceo Inter. Sc. Mat. e Loro Appl., Rome, Accad. Naz. dei Lincei 35 (1977), 1-8.

[Me95] Meylan F., The reflection principle in complex space, Indiana Univ. Math. J. 44 (1995), no. 3, 783-796.

[MMZ01] Meylan, F., Mir, N., Zaitsev, D., Holomorphic extension of smooth CR-mappings between real-analytic and real-algebraic CR-manifolds, preprint, 2001.

[Mi00] Mir, N., On the convergence of formal mappings, to appear in Comm. Anal. Geom., http://arxiv.org/abs/math.CV/0002240 
[Pi75] Pinchuk, S., On the analytic continuation of holomorphic mappings, Math. USSRSb. 27 (1977), 375-392, translation from Mat. Sb. (N.S.) 98 (140), 416-435, 1975.

[Pu90a] Pushnikov, A. Yu., Holomorphicity of CR-mappings into a space of large dimension, Mat. Zametki 48 (1990), no. 3, 147-149.

[Pu90b] Pushnikov, A. Yu., On the holomorphy of CR-mappings of real analytic hypersurfaces, Complex analysis and differential equations, 76-84, Bashkir. Gos. Univ., Ufa, 1990.

[Sh91] Shiffman, B., Separately meromorphic and separately holomorphic functions, Proc. Symp. Pure Math. 52 (1991), 191-198.

[Su92] Sukhov, A., Holomorphic mappings of domains of "wedge" type, Mat. Zametki 52 (1992), no. 3, 141-145, translation in Math. Notes 52 (1993), no. 3-4, 979-982.

[To66] Tomassini, G., Tracce delle functional olomorfe sulle sotto varieta analitiche reali d'una varieta complessa, Ann. Scuola Norm. Sup. Pisa 20 (1966), 31-43.

[Tr86] Trépreau, J.-M., Sur le prolongement holomorphe des fonctions CR définies sur une hypersurface réelle de classe $C^{2}$ dans $\mathbb{C}^{n}$, Invent. Math. 83 (1986), no. 3, 583-592.

[Tu88] Tumanov, A. E., Extension of CR-functions into a wedge from a manifold of finite type, Mat. Sb. (N.S.) 136 (178), no. 1, 128-139 (1988), translation in Math. USSRSb. 64, no. 1, 129-140 (1989).

[Tu90] Tumanov, A.E., Extension of CR-functions into a wedge, Mat. Sb. 181 (1990), no. 7, 951-964, translation in Math. USSR-Sb. 70 (1991), no. 2, 385-398.

[Tu93] Tumanov, A. E. On the propagation of extendibility of CR functions, Complex analysis and geometry (Trento, 1993), 479-498, Lecture Notes in Pure and Appl. Math., 173, Dekker, New York, 1996.

[Tu96] Tumanov, A.E., Analytic discs and the extendibility of CR functions, Integral geometry, Radon transforms and complex analysis (Venice, 1996), 123-141, Lecture Notes in Math., 1684, Springer, Berlin, 1998.

[W78] Webster, S. M., On the reflection principle in several complex variables, Proc. Amer. Math. Soc. 71 (1978), no. 1, 26-28.

[W82] Webster, S. M., Holomorphic mappings of domains with generic corners, Proc. Amer. Math. Soc. 86 (1982), no. 2, 236-240.

[Z99] Zaitsev, D., Algebraicity of local holomorphisms between real-algebraic submanifolds in complex space, Acta Math. 183 (1999), no. 2, 273-305.

Institut de Mathématiques, Université de Fribourg, 1700 Perolles, Fribourg, SWITZERLAND.

E-mail address: francine.meylan@unifr.ch

Université de Rouen, Laboratoire de Mathématiques Raphä̈l Salem, UMR 6085 CNRS, 76821 Mont-Saint-Aignan Cedex, France.

E-mail address: Nordine.Mir@univ-rouen.fr

Mathematisches Institut, Eberhard-Karls-Universität Tübingen, 72076 Tübingen, Germany.

E-mail address: dmitri.zaitsev@uni-tuebingen.de 